\title{
Variáveis ruminais avaliadas por meio de funções matemáticas contínuas
}

\author{
Edenio Detmann ${ }^{(1)}$, Paulo Roberto Cecon(2), Mário Fonseca Paulino(1), Sebastião de Campos Valadares Filho(1), \\ Lara Toledo Henriques ${ }^{(1)}$ e Kelly da Silva Coutinho Detmann ${ }^{(3)}$
}

\begin{abstract}
(1)Universidade Federal de Viçosa (UFV), Dep. de Zootecnia, Av. P.H. Rolfs, s/no, CEP 36571-000 Viçosa, MG. E-mail: detmann@ufv.br, mpaulino@ufv.br, scvfilho@ufv.br, larath@ig.com.br (2)UFV, Dep. de Informática. E-mail: cecon@dpi.ufv.br (3)UFV, Dep. de Biologia Vegetal. E-mail: coutinhokelly@yahoo.com.br
\end{abstract}

\begin{abstract}
Resumo - O objetivo deste trabalho foi avaliar as variáveis $\mathrm{pH}$ e concentração de nitrogênio amoniacal ruminal em bovinos por meio de funções matemáticas contínuas, utilizando-se o polinômio trigonométrico em série de Fourier. Foram utilizados quatro conjuntos de dados simulados referentes à alimentação de vacas em lactação em confinamento e bovinos suplementados em pastejo. Os ajustamentos dos modelos foram realizados por procedimentos iterativos. Os efeitos nutricionais nas diferentes situações de alimentação simuladas foram estimados por operações de cálculo integral. As séries de Fourier apresentam elevada capacidade de ajustamento para descrição do comportamento diário do $\mathrm{pH}$ e concentração de nitrogênio amoniacal ruminal. As variáveis geradas por integração das funções ajustadas apresentam elevada capacidade discriminatória, o que permite refinamentos sobre a interpretação dos efeitos nutricionais de diferentes dietas das características físico-químicas do ambiente ruminal.
\end{abstract}

Termos para indexação: degradação ruminal, nitrogênio amoniacal ruminal, pH ruminal, séries de Fourier.

\section{Rumen variables evaluated through continuum mathematical functions}

\begin{abstract}
The objective of this work was to evaluate rumen $\mathrm{pH}$ and ammonia concentration in cattle through continuum mathematical functions by using the Fourier's trigonometric polynomial. Four simulated dataset, which represent feeding conditions of confined dairy cows and cattle supplemented at pasture were used. The models adjustments were performed by iterative procedures. The nutritional effects in the different simulated feeding situations were evaluated through integral calculus. The Fourier series has showed a good adjustment in describing the daily behavior of rumen $\mathrm{pH}$ and ammonia concentration. Variables obtained through numeric integration show a high discriminatory power, which leads to a fine tuning on the evaluation of diet effects on physical and chemical characteristics of rumen.
\end{abstract}

Index terms: rumen degradation, rumen ammonia nitrogen, rumen $\mathrm{pH}$, Fourier series.

\section{Introdução}

Em estudos sobre nutrição, digestão e metabolismo em ruminantes, a avaliação das características dos processos de degradação ruminal constitui determinante do total de nutrientes disponíveis para o atendimento das necessidades de mantença e produção animal. Entre estas características, o pH e a concentração de nitrogênio amoniacal ruminal (NAR) influenciam diretamente a adequação do meio para a ação e sobrevivência de espécies microbianas específicas (Mould et al., 1983; Hoover, 1986; Russell, 2002) e a disponibilidade de compostos nitrogenados para a síntese de proteína microbiana (Leng, 1990; Soest, 1994).
No entanto, procedimentos experimentais comumente aplicados à avaliação de tais características consideram apenas a mensuração de valores pontuais e, em curto espaço de tempo pós-alimentação (Dias et al., 2000; Detmann et al., 2005), o que pode limitar a compreensão do funcionamento da interação entre o padrão de degradação ruminal e o suprimento de nutrientes ao metabolismo dos animais.

O processo de degradação ruminal e o suprimento de nutrientes a partir do rúmen são eventos contínuos; portanto, o melhor entendimento das relações entre estas características e da adequação do meio ao crescimento microbiano pode ser obtido pela avaliação das variáveis pH e NAR por meio de funções matemáticas contínuas. 
Istasse \& Ørskov (1983), Wedekind et al. (1986) e Kennedy \& Butting (1992) propuseram a interpretação do comportamento do $\mathrm{pH}$ ruminal com enfoque linear, com posterior utilização de fundamentos de cálculo integral. Contudo, a variabilidade temporal de pH e NAR assume comportamento tipicamente não-linear, com taxas de elevação e queda diferenciadas em função das taxas de degradação, de assimilação microbiana, de absorção de produtos da fermentação e de liberação de tampões no rúmen. Além disto, observa-se tendência circadiana do comportamento dessas variáveis de acordo com o padrão da alimentação do animal em condições experimentais.

As séries de Fourier constituem um grupo de modelos não-lineares baseados em funções trigonométricas destinadas à descrição de fenômenos cíclicos (Spiegel, 1976), que possuem capacidade de ajustamento elevada, e que podem ser utilizadas para expressão contínua do comportamento de variáveis como pH e NAR.

$\mathrm{O}$ objetivo deste trabalho foi avaliar as variáveis $\mathrm{pH}$ e concentração de nitrogênio amoniacal ruminais em bovinos, por meio de funções matemáticas contínuas, utilizando-se o polinômio trigonométrico em série de Fourier.

\section{Material e Métodos}

Foram simulados dados relativos a quatro animais: dois estavam relacionados à variável $\mathrm{pH}$ do fluido ruminal e dois, à concentração de NAR (Tabela 1). Valores utilizados neste trabalho se basearam em resultados de $\mathrm{pH}$ ruminal verificados em bovinos confinados, alimentados com dietas com alto nível de concentrado (Cardoso et al., 2000; Dias et al., 2000), em que foram consideradas duas situações teóricas para avaliar a eficiência de ajustamento de uma série de Fourier a diferentes situações de alimentação: com refeições diárias equiidistantes (pH1 - 8 e 20h) e não-equiidistantes ( $\mathrm{pH} 2-8$ e 16h).

Os valores de NAR basearam-se no fornecimento de suplementos protéicos para bovinos manejados a pasto e tiveram como base resultados obtidos em situações brasileiras (Moraes, 2003; Detmann et al., 2005), em que se simulou o fornecimento diário único dos suplementos (10h).

Na conversão das informações pontuais de $\mathrm{pH}$ e NAR em variável contínua, os valores foram submetidos ao ajustamento da função não-linear em série de Fourier no esquema de polinômio trigonométrico (Hsu, 1973; Spiegel, 1976; Thornley \& France, 2005), descrita por:

$$
\mathrm{Y}_{\mathrm{t}}=\mathrm{A}_{0}+\sum_{\mathrm{k}=1}^{\mathrm{K}}\left[\mathrm{A}_{\mathrm{k}} \operatorname{sen}(\mathrm{kct})+\mathrm{B}_{\mathrm{k}} \cos (\mathrm{kct})\right]
$$

em que: $Y_{\mathrm{t}}$ são valores de $\mathrm{pH}$ e NAR $\left(\mathrm{mg} \mathrm{dL}^{-1}\right)$ no momento de amostragem $\mathrm{t} ; \mathrm{A}_{0}$ são estimativas médias de pH e NAR $\left(\mathrm{mg} \mathrm{dL}^{-1}\right) ; \mathrm{A}_{\mathrm{k}}$ e $\mathrm{B}_{\mathrm{k}}$ são parâmetros sem interpretação biológica direta; c é o período fundamental de $\mathrm{pH}$ e NAR $\left(\mathrm{rad} \mathrm{h}^{-1}\right)$; $\mathrm{k}$ é o indexador referente à série de Fourier, sendo inteiro e positivo, variando de 1 a K; e t é o momento de amostragem (horário).

A transformação da informação contínua das variáveis pH e NAR em parâmetros de comparação direta entre animais, denominadas $\mathrm{V}_{\mathrm{pH}}$ e $\mathrm{V}_{\mathrm{NAR}}$ foram conduzidas por meio de integração analítica das funções ajustadas pela equação (1).

Tabela 1. Valores simulados de pH e de concentração de nitrogênio amoniacal (NAR $-\mathrm{mg} \mathrm{dL}^{1}$ ) ruminais.

\begin{tabular}{|c|c|c|c|c|}
\hline \multirow[t]{2}{*}{ Momento de avaliação } & \multicolumn{4}{|c|}{ Situação experimental simulada } \\
\hline & $\overline{\mathrm{pH} 1}$ & $\mathrm{pH} 2$ & NAR1 & NAR2 \\
\hline $\mathrm{Oh}$ & 6,25 & 6,71 & 4,86 & 4,95 \\
\hline $1 \mathrm{~h}$ & 6,52 & 6,75 & 4,88 & 5,20 \\
\hline $2 \mathrm{~h}$ & 6,65 & 6,73 & 5,01 & 5,16 \\
\hline $3 \mathrm{~h}$ & 6,71 & 6,79 & 4,92 & 5,12 \\
\hline $4 \mathrm{~h}$ & 6,80 & 6,81 & 4,96 & 4,99 \\
\hline $5 \mathrm{~h}$ & 6,84 & 6,85 & 4,99 & 5,03 \\
\hline $6 \mathrm{~h}$ & 6,87 & 6,82 & 5,03 & 5,09 \\
\hline $7 \mathrm{~h}$ & 6,85 & 6,82 & 4,99 & 5,00 \\
\hline $8 \mathrm{~h}$ & $6,86^{(1)}$ & $6,81^{(1)}$ & 5,09 & 5,14 \\
\hline $9 \mathrm{~h}$ & 6,32 & 6,23 & 5,14 & 5,13 \\
\hline $10 \mathrm{~h}$ & 5,98 & 5,89 & $5,15^{(1)}$ & $5,12^{(1)}$ \\
\hline $11 \mathrm{~h}$ & 6,02 & 5,99 & 8,75 & 9,13 \\
\hline $12 \mathrm{~h}$ & 6,09 & 6,05 & 15,23 & 17,96 \\
\hline $13 \mathrm{~h}$ & 6,25 & 6,17 & 21,85 & 23,26 \\
\hline $14 \mathrm{~h}$ & 6,58 & 6,40 & 20,53 & 22,15 \\
\hline $15 \mathrm{~h}$ & 6,67 & 6,68 & 18,50 & 19,46 \\
\hline $16 \mathrm{~h}$ & 6,72 & $6,69^{(1)}$ & 16,90 & 19,03 \\
\hline $17 \mathrm{~h}$ & 6,73 & 6,21 & 14,83 & 15,20 \\
\hline $18 \mathrm{~h}$ & 6,75 & 5,99 & 13,92 & 13,05 \\
\hline $19 \mathrm{~h}$ & 6,76 & 6,03 & 12,56 & 11,36 \\
\hline $20 \mathrm{~h}$ & $6,85^{(1)}$ & 6,19 & 10,86 & 9,54 \\
\hline $21 \mathrm{~h}$ & 6,15 & 6,34 & 8,92 & 7,52 \\
\hline $22 \mathrm{~h}$ & 5,90 & 6,47 & 6,23 & 6,98 \\
\hline $23 \mathrm{~h}$ & 6,05 & 6,62 & 6,01 & 5,85 \\
\hline $24 \mathrm{~h}$ & 6,17 & 6,65 & 5,50 & 5,15 \\
\hline
\end{tabular}

${ }^{(1)}$ Valores correspondentes ao momento de fornecimento da dieta ou suplemento. 
Em estudos nos quais investiga-se a influência do pH ruminal sobre as características de degradação ruminal, normalmente estabelecem-se patamares mínimos, a partir dos quais passa-se a comprometer a atividade microbiana, notadamente sobre a degradação dos carboidratos fibrosos (Mould et al., 1983; Hoover, 1986). Desta forma, definese $\mathrm{V}_{\mathrm{pH}}$ como a área abrangida pela função ajustada que se situa abaixo do patamar mínimo requerido para a atividade adequada dos microrganismos ruminais.

Define-se matematicamente $\mathrm{V}_{\mathrm{pH}}$, cuja unidade é definida pelo produto entre $\mathrm{pH}$ e tempo $(\mathrm{h})$, por:

$V_{p H}=\left[\int_{t_{1}}^{t_{r_{1}}} C_{p H} d t-\int_{t_{1}}^{t_{t_{1}}} f(t) d t\right]+\ldots+\left[\int_{t_{1}}^{t_{r}} C_{p H} d t-\int_{t_{1}}^{t_{r}} f(t) d t\right]$

em que $\mathrm{C}_{\mathrm{pH}}$ é o valor constante de $\mathrm{pH}$ ruminal, o qual exprime o valor de patamar mínimo; $\mathrm{f}(\mathrm{t})$ é a função ajustada para o pH ruminal (equação 1); e $t_{i}$ e $t_{i}$, são valores de tempo equivalentes ao $\mathrm{C}_{\mathrm{pH}}$ entre os quais encontra-se $\mathrm{V}_{\mathrm{pH}}$ (horário).

Em razão de o $\mathrm{C}_{\mathrm{pH}}$ ser constante, sua integração numérica se dá pela equação:

$$
\int_{\mathrm{t}_{\mathrm{i}}}^{\mathrm{t}_{\mathrm{i}}} \mathrm{C}_{\mathrm{pH}} \mathrm{dt}=\mathrm{C}_{\mathrm{pH}} \times\left(\mathrm{t}_{\mathrm{i}^{\prime}}-\mathrm{t}_{\mathrm{i}}\right)
$$

A influência da concentração de NAR é normalmente investigada em patamares mínimos para manutenção da atividade microbiana ruminal ou maximização de características de consumo e digestão (Satter \& Slyter, 1974; Leng, 1990). Define-se $\mathrm{V}_{\mathrm{NAR}}$ como a área abrangida pela função ajustada que se situa acima do patamar mínimo definido para que tais características sejam estabelecidas.

Define-se matematicamente $\mathrm{V}_{\mathrm{NAR}}$, cuja unidade é definida pelo produto entre NAR ( $\left.\mathrm{mg} \mathrm{dL}^{-1}\right)$ e tempo (h), por:

$$
V_{N A R}=\left[\int_{t_{1}}^{t_{1}} f(t) d t-\int_{t_{1}}^{t_{1}} C_{N A R} d t\right]+\ldots+\left[\int_{t_{1}}^{t_{P}} f(t) d t-\int_{t_{1}}^{t_{1}} C_{N A R} d t\right]
$$

em que $\mathrm{C}_{\mathrm{NAR}}$ é o valor constante de NAR $\left(\mathrm{mg} \mathrm{dL}^{-1}\right)$, o qual exprime o valor de patamar mínimo; $\mathrm{f}(\mathrm{t})$ é função ajustada para a concentração de NAR (equação 1); e $t_{i}$ e $t_{i}$ ' são valores de tempo equivalentes a $C_{\text {NAR }}$ entre os quais encontra-se $\mathrm{V}_{\mathrm{NAR}}$ (horário).

De forma similar, a integração numérica de $\mathrm{C}_{\mathrm{NAR}}$ se dá pela equação: $\int_{\mathrm{t}_{\mathrm{i}}}^{\mathrm{t}_{\mathrm{i}}} \mathrm{C}_{\mathrm{NAR}} \mathrm{dt}=\mathrm{C}_{\mathrm{NAR}} \times\left(\mathrm{t}_{\mathrm{i}^{\prime}}-\mathrm{t}_{\mathrm{i}}\right)$

A integral de f(t) (equação 1) é dada por (Shenk, 1985):

$\int_{t_{i}}^{t_{i j}} f(t) d t=\left.\left\{A_{0} t+\sum_{k=1}^{K}\left[-A_{k} \cos (k c t)+B_{k} \operatorname{sen}(k c t)\right]\right\}\right|_{t_{i}} ^{t_{i t}}$

Todos os ajustamentos não-lineares foram conduzidos por meio do algoritmo iterativo de Gauss-Newton (Souza, 1998), utilizando-se o PROC NLIN do SAS (Statistical Analysis System). Os valores de $t_{i}$ e $t_{i}$, foram obtidos por procedimentos iterativos.

A qualidade do ajustamento das equações não-lineares foi avaliada por intermédio do desvio-padrão assintótico, do coeficiente de determinação, do número de corridas de sinal e da dispersão de resíduos padronizados (Draper \& Smith, 1966; Bard, 1974; Souza, 1998).

Os valores de desvios-padrão assintóticos foram estimados pelo método da máxima verossimilhança:

$$
D P A=\sqrt{\frac{\sum_{i}^{I} \hat{e}_{i}^{2}}{n}}=\sqrt{\frac{\sum_{i}^{I}\left(y_{i}-\hat{y}_{i}\right)^{2}}{n}}
$$

em que DPA é o desvio-padrão assintótico; $\hat{e}_{i}$ é o erro relativo à observação $\mathrm{i} ; \mathrm{y}_{\mathrm{i}}$ é o valor observado $\mathrm{i} ; \hat{y}_{\mathrm{i}}$ é o valor estimado $i$; e n é o número de observações.

Os resíduos padronizados foram obtidos a partir da relação:

$$
\mathrm{RP}_{\mathrm{i}}=\frac{\hat{\mathrm{e}}_{\mathrm{i}}}{\mathrm{DPA}}=\frac{\mathrm{y}_{\mathrm{i}}-\hat{\mathrm{y}}_{\mathrm{i}}}{\mathrm{DPA}}
$$

em que $\mathrm{RP}_{\mathrm{i}}$ é o resíduo padronizado $\mathrm{i}$.

$\mathrm{O}$ coeficiente de determinação foi estimado como o quadrado do coeficiente de correlação entre valores preditos e observados da variável dependente (Souza, 1998).

A dimensão da série de Fourier, determinada pelo indexador $\mathrm{k}$, foi definida de acordo com os indicadores de qualidade de ajustamento acima definidos. Em ajustamentos não-lineares, adotou-se 0,05 como nível crítico de probabilidade assintótica para o erro tipo I.

\section{Resultados e Discussão}

Estatísticas descritivas de ajustamento das séries de Fourier às diferentes sequiências de dados simulados são apresentadas na Tabela 2. 
Em todas as situações avaliadas, observaram-se elevados coeficientes de determinação e baixos desvios-padrão assintóticos, o que indica boa qualidade de ajustamento das séries de Fourier aos conjuntos de dados simulados. Embora a situação $\mathrm{pH} 1$ tenha apresentado coeficiente de determinação pouco inferior aos demais (Tabela 2), ressaltase que a ampliação da série (equação 1) não implicou em elevação desta estatística.

$\mathrm{Na}$ situação $\mathrm{pH} 2$, verificou-se necessidade do estabelecimento de série de Fourier superior às demais situações, particularmente em comparação a pH1 (Tabela 2). Este fato pode ser atribuído à característica não-eqüidistante do manejo de alimentação, que implica, em condições práticas, em padrão de estabelecimento do $\mathrm{pH}$ ruminal de forma diferenciada, com efeitos marcantes da primeira refeição diária no momento de tornar disponível a segunda refeição. Contudo, mesmo com esta característica de assimetria, verificou-se alta capacidade de ajustamento da série de Fourier.

As corridas de sinal permitem indicar o comportamento da função ajustada de acordo com o conjunto de dados observados. Baixo número de corridas de sinal indica que podem ocorrer regiões com sub ou superestimativas da variável dependente (López et al., 1999; Detmann et al., 2001). Em todas as situações avaliadas, observou-se relação aproximada de uma corrida de sinal para cada três ou quatro observações, o que pode ser considerado adequado pela elevada complexidade do ciclo circadiano das variáveis avaliadas.

Tabela 2. Estatísticas descritivas relativas ao ajustamento das séries de Fourier e estimativas de parâmetros do modelo não linear.

\begin{tabular}{|c|c|c|c|c|}
\hline \multirow[t]{2}{*}{ Estatística } & \multicolumn{4}{|c|}{ Situação simulada } \\
\hline & $\mathrm{pH} 1$ & $\mathrm{pH} 2$ & NAR1 & NAR2 \\
\hline $\mathrm{DPA}^{(1)}$ & 0,140 & 0,085 & 1,586 & 1,584 \\
\hline $\mathrm{R}^{2}$ & 0,8501 & 0,9218 & 0,9178 & 0,9316 \\
\hline Corridas de sinal & 6 & 10 & 7 & 7 \\
\hline \multicolumn{5}{|l|}{ Parâmetro $^{(2)}$} \\
\hline $\mathrm{A}_{0}$ & 6,4982 & 6,4444 & 9,8825 & 10,4187 \\
\hline $\mathrm{A}_{1}$ & 0,0641 & 0,2524 & $-6,7801$ & $-7,9732$ \\
\hline $\mathrm{B}_{1}$ & 0,0131 & 0,1500 & $-2,6330$ & $-1,3993$ \\
\hline $\mathrm{A}_{2}$ & 0,2487 & 0,1765 & 2,3339 & 2,1180 \\
\hline $\mathrm{B}_{2}$ & $-0,3273$ & $-0,1712$ & $-1,1281$ & $-2,4287$ \\
\hline $\mathrm{A}_{3}$ & - & $-0,0443$ & - & - \\
\hline $\mathrm{B}_{3}$ & - & 0,2223 & - & - \\
\hline $\mathrm{c}$ & 0,2546 & 0,2775 & 0,2836 & 0,3039 \\
\hline
\end{tabular}

${ }^{(1)}$ Desvio-padrão assintótico. ${ }^{(2)}$ Ver equação 1 para mais detalhes.
A inspeção do padrão de comportamento dos resíduos padronizados (Figura 1) corrobora o diagnóstico de bom ajustamento das séries de Fourier ao conjunto de dados. De forma geral, espera-se que o conjunto de resíduos demonstre padrão de dispersão regular em torno do eixo das abscissas, o qual representa a variável independente (Draper \& Smith, 1966), como observado (Figura 1). Aproximadamente 70 e $95 \%$ dos resíduos concentraramse dentro das faixas equivalentes a uma e duas unidades de desvio-padrão assintótico, freqüência que pode ser considerada satisfatória ao perfil de ajustamento nãolinear proposto.

A existência de um período fundamental para uma série periódica de Fourier é definida pela condição (Spiegel, 1976):

$\mathrm{f}(\mathrm{t}+\mathrm{P})=\mathrm{f}(\mathrm{t}) ; \forall \mathrm{t}$

em que: P é o período fundamental (horas).

No caso específico do polinômio trigonométrico expresso pela equação 1 , pressupondo-se valores de $\mathrm{k}$ inteiros e positivos, estima-se o período fundamental como (Hsu, 1973; Spiegel, 1976):

$\mathrm{P}=\frac{2 \pi}{\mathrm{Kc}}$

A partir dos resultados expressos na Tabela 2, períodos fundamentais de 12,79, 7,55, 11,08 e 10,34 horas, para pH1, pH2, NAR1 e NAR2, respectivamente, são estimados.

A ilustração do período fundamental pode ser realizada com base no conjunto de dados pH1, demonstrado na

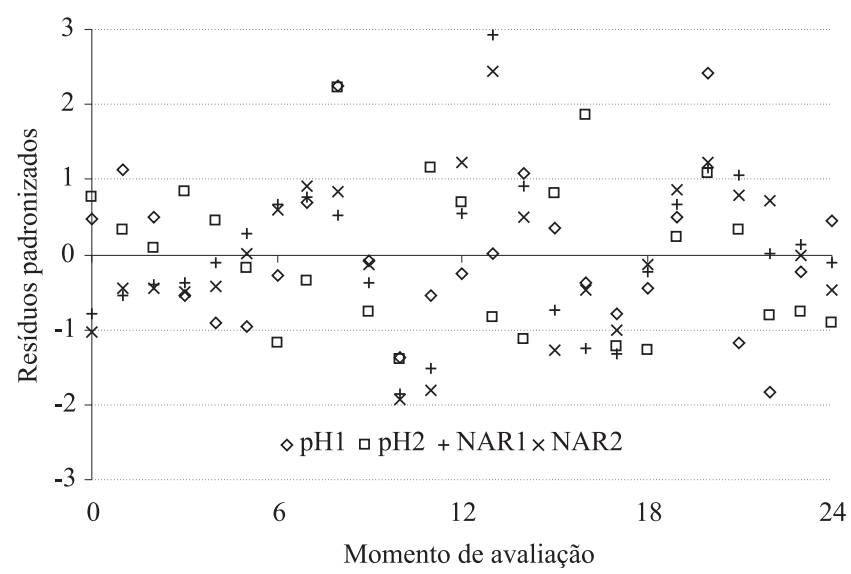

Figura 1. Dispersão gráfica dos resíduos padronizados de acordo com o momento de avaliação (horário). 
Figura 2. Observa-se que a função assume o valor estabelecido de $\mathrm{C}_{\mathrm{pH}}$, por aproximação a partir de valores inferiores a este, próximo a $1 \mathrm{~h} 40$. Tal comportamento apenas irá se repetir às $14 \mathrm{~h} 20$. Desta forma, o ciclo aproxima-se do valor estimado de período fundamental descrito.

A dimensão do período fundamental seria de aproximadamente 24 horas em situações que envolvessem evento de distúrbio único, como a suplementação em horário único para animais em pastejo, ou de 12 horas em situações de duplo distúrbio, como a alimentação de animais estabulados em refeições equiidistantes.

Somente a situação simulada $\mathrm{pH} 1$ aproximou-se da definição teórica. Contudo, a heterogeneidade dos ciclos fundamentais para as demais situações simuladas não deve ser vista como inadequação das séries de Fourier em se ajustar aos valores observados. Isto reflete a complexidade do comportamento das variáveis estudadas de acordo com características inerentes a cada animal e situação alimentar em relação às taxas de degradação
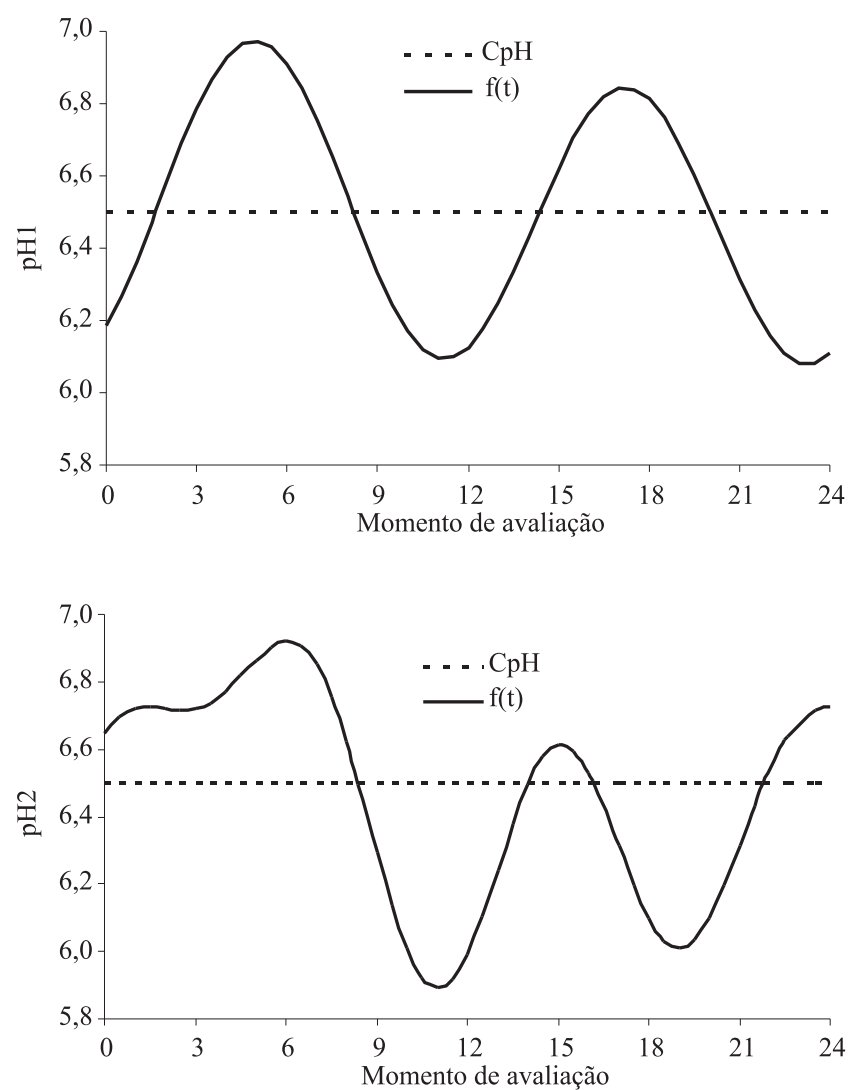

Figura 2. Função ajustada para o $\mathrm{pH}$ ruminal [f(t)] e limite do platô de avaliação $\left(\mathrm{C}_{\mathrm{pH}}\right)$ de acordo com a situação simulada. de substratos, de assimilação e crescimento microbiano, de remoção de produtos de fermentação do meio e de tamponamento. As estimativas de $\mathrm{V}_{\mathrm{pH}}$ e $\mathrm{V}_{\mathrm{NAR}}$, bem como os intervalos críticos de tempo são apresentadas na Tabela 3.

$\mathrm{Na}$ avaliação de $\mathrm{V}_{\mathrm{pH}}$ estabeleceu-se como $\mathrm{C}_{\mathrm{pH}} \mathrm{O}$ valor de 6,5. Foram observadas três áreas localizadas abaixo de $\mathrm{C}_{\mathrm{pH}}$ para a situação $\mathrm{pH} 1$ e duas áreas para $\mathrm{pH} 2$ (Figura 2), cujo somatório resultou em valores de 0,0212 e 0,6317 , respectivamente.

A interpretação direta desses valores indica a ponderação entre a intensidade de ocorrência do $\mathrm{pH}$ ruminal em valores abaixo de limites considerados prejudiciais à atividade microbiana ruminal (neste caso definido arbitrariamente como 6,5) e o tempo no qual isto ocorre. Desta forma, a situação pH2 conferiu ambiente não-propício em termos de $\mathrm{pH}$ ruminal de forma mais intensa que pH1 (Tabela 3).

Embora em ambas as situações verifiquem-se valores pontuais similares de $\mathrm{pH}$ ruminal (Tabela 1), a avaliação de $\mathrm{V}_{\mathrm{pH}}$ indicou que as situações simuladas diferem na formação de ambientes não-propícios ao crescimento microbiano, principalmente microganismos fibrolíticos. Isto reflete o maior tempo para liberação de tampões e remoção de produtos de fermentação entre refeições simulado em $\mathrm{pH} 1$, ao passo que o efeito residual da primeira refeição diária parece ser ainda intenso em $\mathrm{pH} 2$ no momento da segunda refeição (Figura 2), o que pode ser indiretamente avaliado pela menor dimensão do período fundamental observado para $\mathrm{pH} 2$ (Tabela 2).

Embora existam avaliações por meio de aproximações geométricas ou lineares da estimação de $\mathrm{V}_{\mathrm{pH}}$ (Istasse \& Ørskov, 1983; Wedekind et al., 1986; Kennedy \&

Tabela 3. Limites de tempo nos quais ocorreram rompimentos do limite de platô e estimativas das variáveis relacionadas ao $\mathrm{pH}\left(\mathrm{V}_{\mathrm{pH}}\right)$ e concentração de nitrogênio amoniacal ruminal $\left(\mathrm{V}_{\mathrm{NAR}}\right)$ de acordo com diferentes situações simuladas.

\begin{tabular}{cccr}
\hline Situação simulada & \multicolumn{2}{c}{ Limites de tempo } & $\mathrm{V}_{\mathrm{pH}} / \mathrm{V}_{\mathrm{NAR}}$ \\
\cline { 2 - 3 } & \multicolumn{1}{c}{$\mathrm{t}_{\mathrm{i}}$} & $\mathrm{t}_{\mathrm{i}}{ }^{\prime}$ & \\
\hline $\mathrm{pH} 1$ & 0,00 & 1,65 & 0,0030 \\
& 8,20 & 14,35 & 0,0111 \\
& 20,05 & 24,00 & 0,0071 \\
\hline Total & - & - & 0,0212 \\
\hline $\mathrm{pH} 2$ & 8,40 & 14,00 & 0,3115 \\
& 16,15 & 21,80 & 0,3202 \\
\hline Total & - & - & 0,6317 \\
\hline NAR1 & 10,64 & 19,58 & 15,66 \\
NAR2 & 10,50 & 19,00 & 22,40 \\
\hline
\end{tabular}


Butting, 1992), a interpretação dessa variável por intermédio de funções contínuas fornece, dentro de princípios probabilísticos, a projeção do comportamento da variável para todo e qualquer momento em todo o ciclo de avaliação, incluindo-se os intervalos entre pontos amostrais. Em adição, a versatilidade de ajustamento das séries de Fourier (Tabela 2), além de propiciar valores cíclicos relevantes do ponto de vista biológico, com baixa falta de ajustamento, confere padrões de interpretação adicionais, como as estimativas dos períodos fundamentais.

A interpretação contínua de variáveis, como o $\mathrm{pH}$ ruminal, pode conduzir a inferências mais seguras sobre os efeitos da dieta nos padrões físico-químicos do ambiente ruminal. Embora interpretações pontuais, em períodos imediatamente pós-alimentação, sejam comuns em procedimentos experimentais com ruminantes, em alguns casos, podem conduzir a sofismas sobre os verdadeiros efeitos dietéticos.

Tomando-se como base as situações pH1 e pH2, percebe-se que em ambas houve comportamento similar do pH ruminal após a primeira refeição (8h) (Figura 2). Contudo, a avaliação do ciclo total permite evidenciar que em $\mathrm{pH} 2$ verificou-se ocorrência de ambientes nãopropícios a atividades microbiana de forma mais intensa (Tabela 3). Logo, a avaliação pontual em pequenos intervalos de tempo pós-alimentação matinal poderia levar a conclusões errôneas sobre a manutenção de condições adequadas para a atividade dos microrganismos no rúmen durante todo o dia.

Apesar de constituir variável comum em estudos ruminais, não foram encontrados dados sobre a avaliação da concentração de NAR por meio de funções matemáticas contínuas. Contudo, observou-se, de forma similar ao $\mathrm{pH}$ ruminal, boa adequação das séries de Fourier em descrever o comportamento dessa variável (Tabela 2 e Figura 3).

De forma inversa ao $\mathrm{pH}$ ruminal, a concentração de NAR tem como base comum o estabelecimento de níveis mínimos para adequação do meio de crescimento à disponibilidade de compostos nitrogenados para o anabolismo microbiano (Leng, 1990; Russell, 2002). Dessa forma, nas avaliações conduzidas estabeleceuse o valor de $\mathrm{C}_{\mathrm{NAR}}$ em $10 \mathrm{mg} \mathrm{dL}^{-1}$ com base nas recomendações de Leng (1990) e Sampaio (2007).

$\mathrm{A}$ interpretação direta de $\mathrm{V}_{\mathrm{NAR}}$ (Tabela 3) permite a ponderação da ocorrência de valores de concentração de NAR de acordo com a intensidade (valor absoluto) e
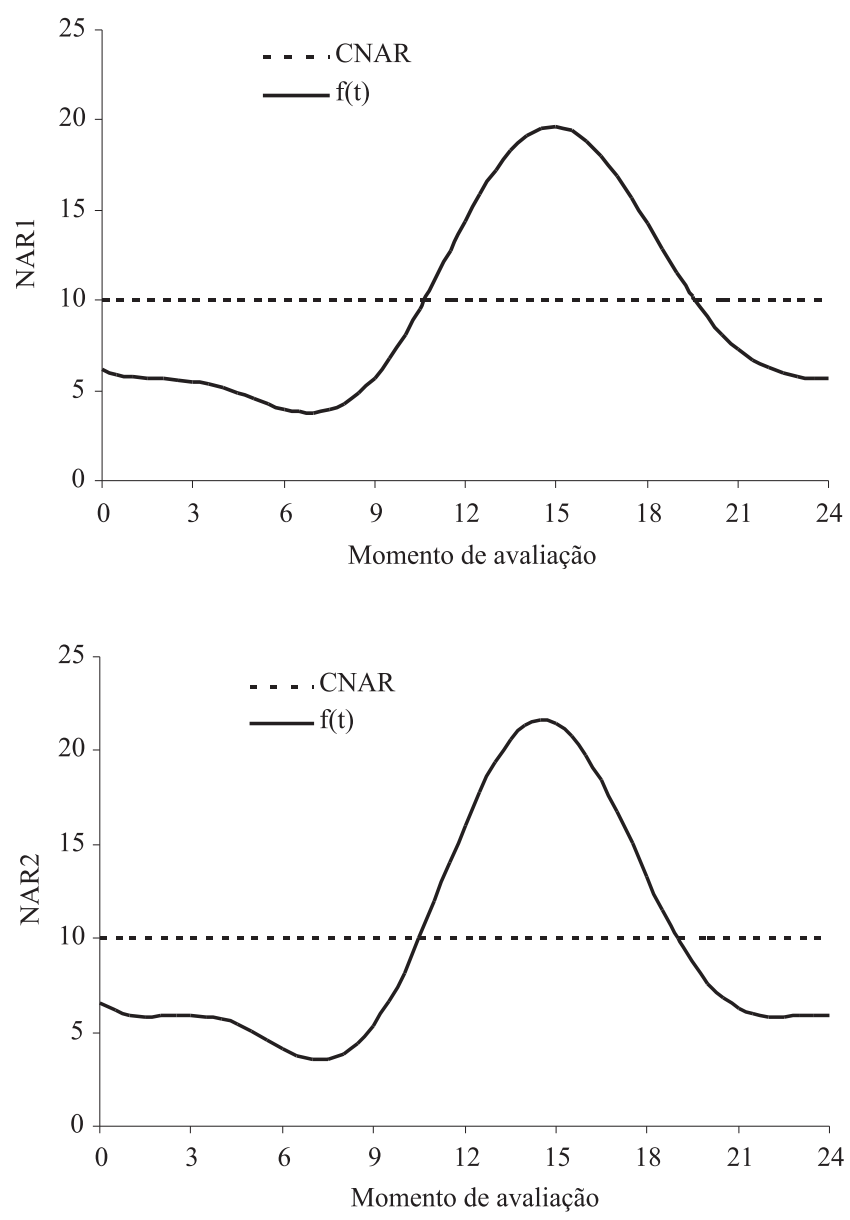

Figura 3. Função ajustada para a concentração de nitrogênio amoniacal ruminal $[\mathrm{f}(\mathrm{t})]$ e limite do platô de avaliação $\left(\mathrm{C}_{\mathrm{NAR}}\right)$ de acordo com a situação simulada.

o tempo no qual tais valores são mantidos acima do patamar mínimo estabelecido $\left(\mathrm{C}_{\mathrm{NAR}}\right)$.

Os resultados deste trabalho contêm evidências do elevado poder de discriminação associado à nova variável produzida, neste caso, $\mathrm{V}_{\mathrm{NAR}}$ (Figura 3), além dos aspectos discutidos sobre o $\mathrm{pH}$ ruminal. Apesar de as informações pontuais obtidas após a suplementação serem aparentemente similares (Tabela 1), observa-se que em NAR2 ocorre maior intensidade de estabelecimento de valores adequados, uma vez que houve estimativa de $\mathrm{V}_{\mathrm{NAR}}$ maior (Tabela 3 ).

\section{Conclusão}

As series de Fourier constituem instrumentos adequados de descrição das variáveis $\mathrm{pH}$ e 
concentração de nitrogênio amoniacal ruminais como funções contínuas em relação ao tempo e permitem a identificação e maior discriminação de características dietéticas na adequação físico-química do ambiente ruminal.

\section{Referências}

BARD, Y. Nonlinear parameter estimation. New York: Academic Press, 1974. 341p.

CARDOSO, R.C.; VALADARES FILHO, S.C.; SILVA, J.F.C da; PAULINO, M.F.; VALADARES, R.F.D.; CECON, P.R.; COSTA, M.A.L. Síntese microbiana, $\mathrm{pH}$ e concentração de amônia ruminal $\mathrm{e}$ balanço de compostos nitrogenados, em novilhos $\mathrm{F}_{1}$ Limousin $\mathrm{x}$ Nelore. Revista Brasileira de Zootecnia, v.29, p.1844-1852, 2000.

DETMANN, E.; CECON, P.R.; PAULINO, M.F.; ZERVOUDAKIS, J.T.; VALADARES FILHO, S.C.; ARAÚJO, C.V. Estimação de parâmetros da cinética de trânsito de partículas em bovinos sob pastejo por diferentes sequiências amostrais. Revista Brasileira de Zootecnia, v.30, p.222-230, 2001.

DETMANN, E.; PAULINO, M.F.; VALADARES FILHO, S.C.; CECON, P.R.; ZERVOUDAKIS, J.T.; CABRAL, L.S.; GONÇALVES, L.C.; VALADARES, R.F.D. Níveis de proteína em suplementos para terminação de bovinos em pastejo durante o período de transição seca/águas: digestibilidade aparente e parâmetros do metabolismo ruminal e dos compostos nitrogenados. Revista Brasileira de Zootecnia, v.34, p.1380-1391, 2005.

DIAS, H.L.C.; VALADARES FILHO, S.C.; SILVA, J.F.C. da; PAULINO, M.F.; CECON, P.R.; VALADARES, R.F.D.; RENNÓ, L.N.; COSTA, M.A.L. Eficiência de síntese microbiana, pH e concentrações ruminais de amônia em novilhos $F_{1}$ Limousin $x$ Nelore alimentados com dietas contendo cinco níveis de concentrado. Revista Brasileira de Zootecnia, v.29, p.555-563, 2000.

DRAPER, N.R.; SMITH, H. Applied regression analysis. New York: John Willey \& Sons, 1966. 407p.

HOOVER, W.H. Chemical factors involved in ruminal fiber digestion. Journal of Dairy Science, v.69, p.2755-2766, 1986.

HSU, H.P. Análise de Fourier. Rio de Janeiro: LTC Editora, 1973. $274 \mathrm{p}$.

ISTASSE, L.; ØRSKOV, E.R. The correlation between extent of $\mathrm{pH}$ depression and degradability of washed hay in sheep given hay and concentrate. Proceedings of Nutrition Society, v.42, p.32A, 1983.
KENNEDY, D.W.; BUNTING, L.D. Effects of starch on ruminal fermentation and detergent fibre digestion in lambs fed bermudagrass hay. Animal Feed Science and Technology, v.36, p.91-100, 1992. LENG, R.A. Factors affecting the utilization of "poor-quality" forages by ruminants particularly under tropical conditions. Nutrition Research Review, v.3, p.277-303, 1990.

LÓPEZ, S.; FRANCE, J.; DHANOA, M.S.; MOULD, F.; DJKISTRA, J. Comparison of mathematical models to describe disappearance curves obtained using the polyester bag technique for incubating feeds in the rumen. Journal of Animal Science, v.77, p.1875-1888, 1999.

MORAES, E.H.B.K. Suplementos múltiplos para recria e terminação de novilhos mestiços em pastejo durante os períodos de seca e transição seca-águas. 2003. 60p. Dissertação (Mestrado) - Universidade Federal de Viçosa, Viçosa.

MOULD, F.L.; ØRSKOV, E.R.; MANNS, O. Associative effects of mixed feeds. I. Effects of type and level of supplementation and the influence of the rumen $\mathrm{pH}$ on cellulolysis in vivo and dry matter digestion of various roughages. Animal Feed Science and Technology, v.10, p.15-30, 1983.

RUSSELL, J.B. Rumen microbiology and its role in ruminant nutrition. Ithaca: James B. Russell, 2002. 119p.

SAMPAIO, C.B. Consumo, digestibilidade e dinâmica ruminal em bovinos alimentados com forragem tropical de baixa qualidade suplementados com compostos nitrogenados. 2007. 53p. Dissertação (Mestrado) - Universidade Federal de Viçosa, Viçosa.

SATTER, L.D.; SLYTER, L.L. Effect of ammonia concentration on rumen microbial protein production in vitro. British Journal of Nutrition, v.32, p.199-208, 1974.

SHENK, A. Cálculo e geometria analítica. Volume 1. 2 ed. Rio de Janeiro: Campus, 1985. 496p.

SOEST, P.J. van. Nutritional ecology of the ruminant. 2.ed. Ithaca: Cornell University Press, 1994. 476p.

SOUZA, G.S. Introdução aos modelos de regressão linear e não-linear. Brasília: Embrapa-SPI, 1998. 505p.

SPIEGEL, M.R. Análise de Fourier. São Paulo: McGraw-Hill do Brasil, 1976. 249p.

THORNLEY, J.H.M.; FRANCE, J. Mathematical model in agriculture: quantitative methods for the plant, animal and ecological sciences. Wallingford: CAB International, 2005. 906p.

WEDEKIND, K.J.; MUNTIFERING, R.B.; BARKER, K.B. Effects of diet concentrate level and sodium bicarbonate on site and extent of forage fiber digestion in the gastrointestinal tract of wethers. Journal of Animal Science, v.62, p.1388-1395, 1986. 